\title{
Erratum to: The Efficacy and Safety of Tolvaptan in Patients with Hyponatremia: A Meta-Analysis of Randomized Controlled Trials
}

Benlei Li ${ }^{1} \cdot$ Dong Fang ${ }^{1} \cdot$ Cheng Qian $^{1} \cdot$ Hongliang Feng $^{2} \cdot$ Yanggan Wang $^{1}$

Published online: 22 February 2017

(C) Springer International Publishing Switzerland 2017

\section{Erratum to: Clin Drug Investig \\ DOI 10.1007/s40261-016-0470-3}

In the original publication, Figs. 3, 4, 5 and 6 were labelled incorrectly. Below are the correct versions of Figs. 3, 4, 5 and 6:

The online version of the original article can be found under doi:10.1007/s40261-016-0470-3.

Yanggan Wang

wb000813@whu.edu.cn

1 Department of Cardiology, Zhongnan Hospital of Wuhan University, No. 169 Donghu Road, Wuhan 430071, Hubei, China

2 Department of Neurology, Zhongnan Hospital of Wuhan University, No. 169 Donghu Road, Wuhan 430071, Hubei, China 


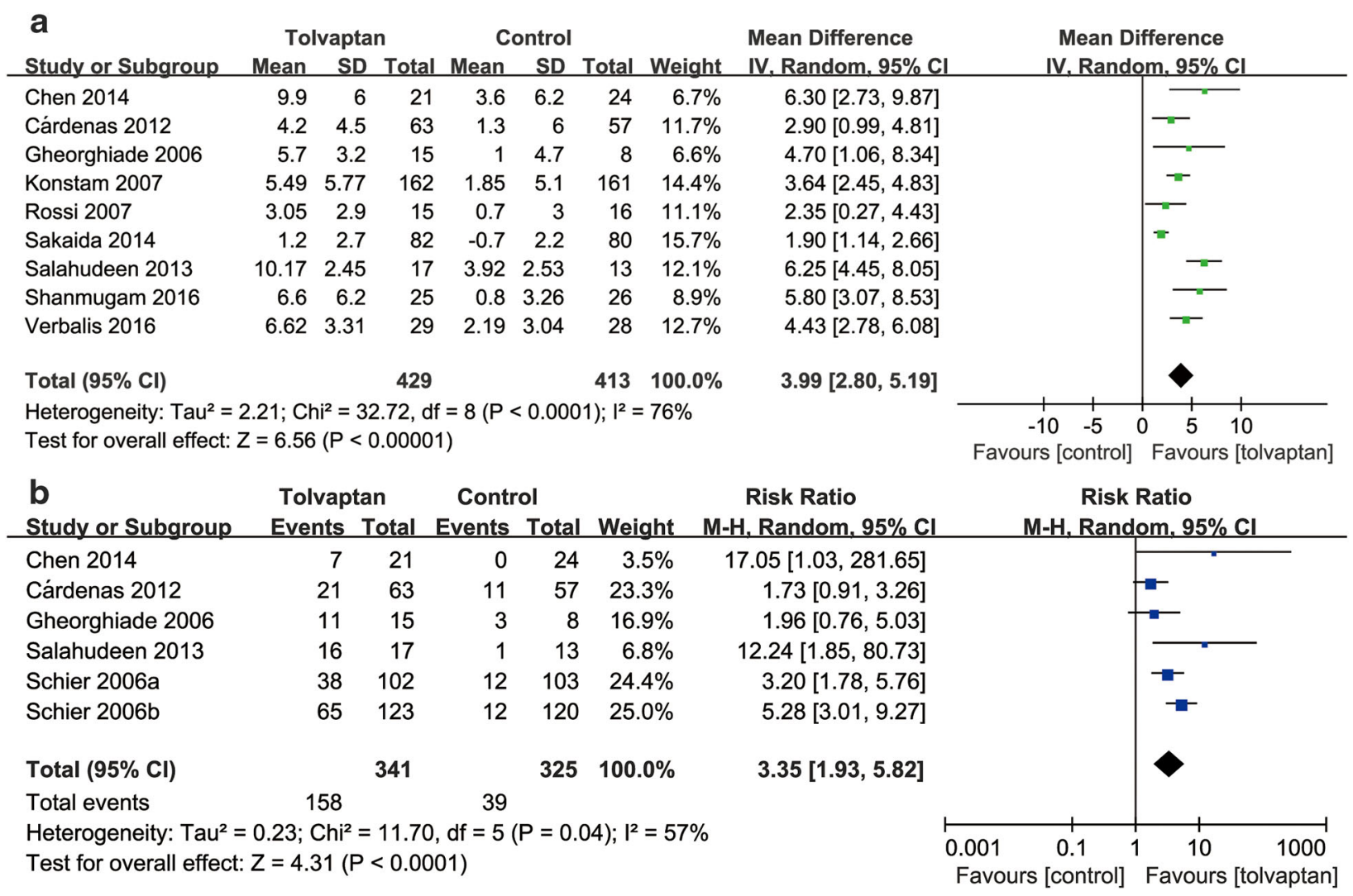

Fig. 3 Effect of tolvaptan on changes in serum sodium concentrations (a) and serum sodium correction rates (b) in patients with hyponatremia. $C I$ confidence interval, $d f$ degrees of freedom, $I V$ inverse variance, $M-H$ Mantel-Haenszel, $S D$ standard deviation

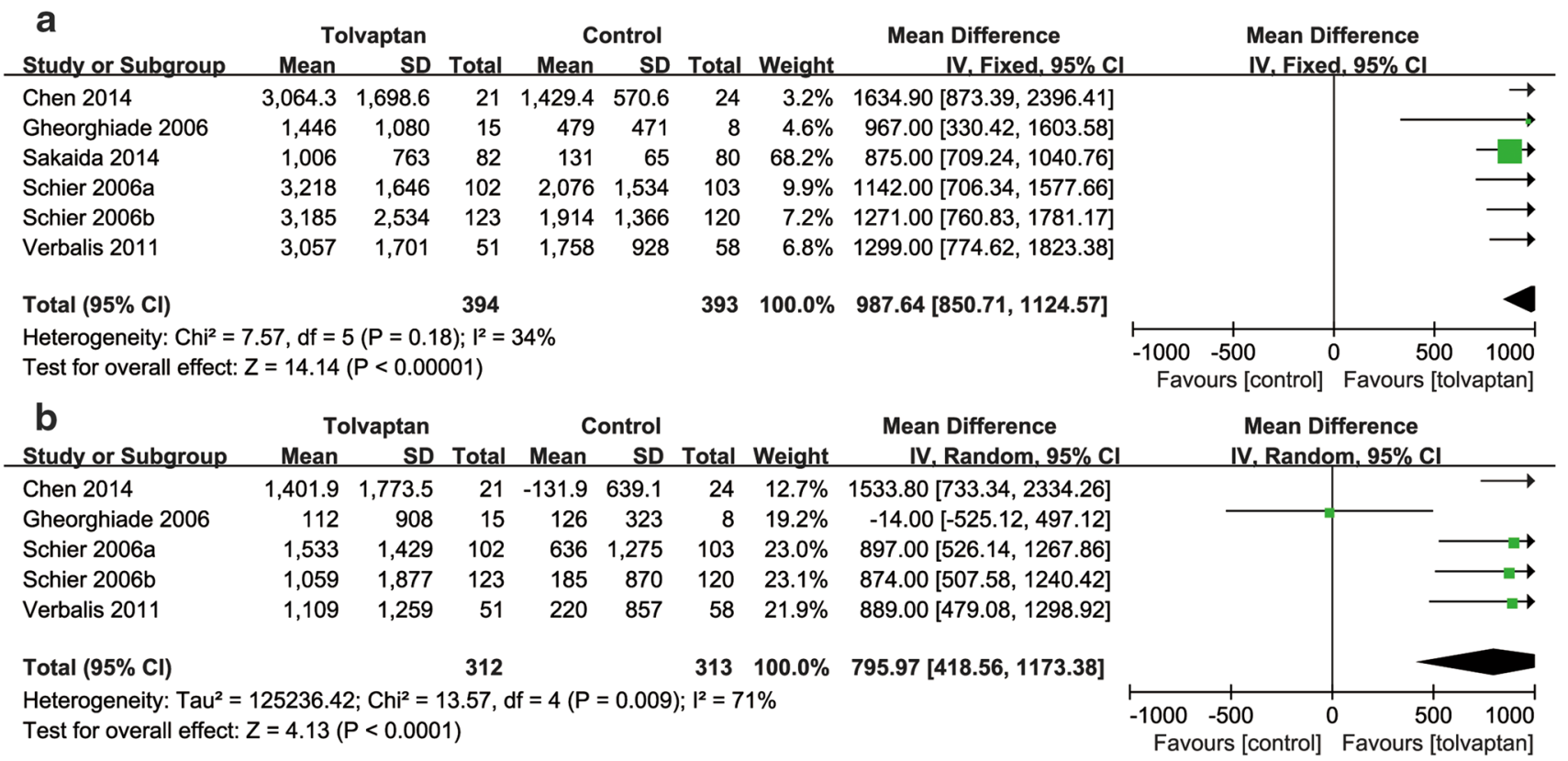

Fig. 4 Effect of tolvaptan on 24-h urine output (a) and net fluid balance (b) in patients with hyponatremia. $C I$ confidence interval, $d f$ degrees of freedom, $I V$ inverse variance, $S D$ standard deviation 


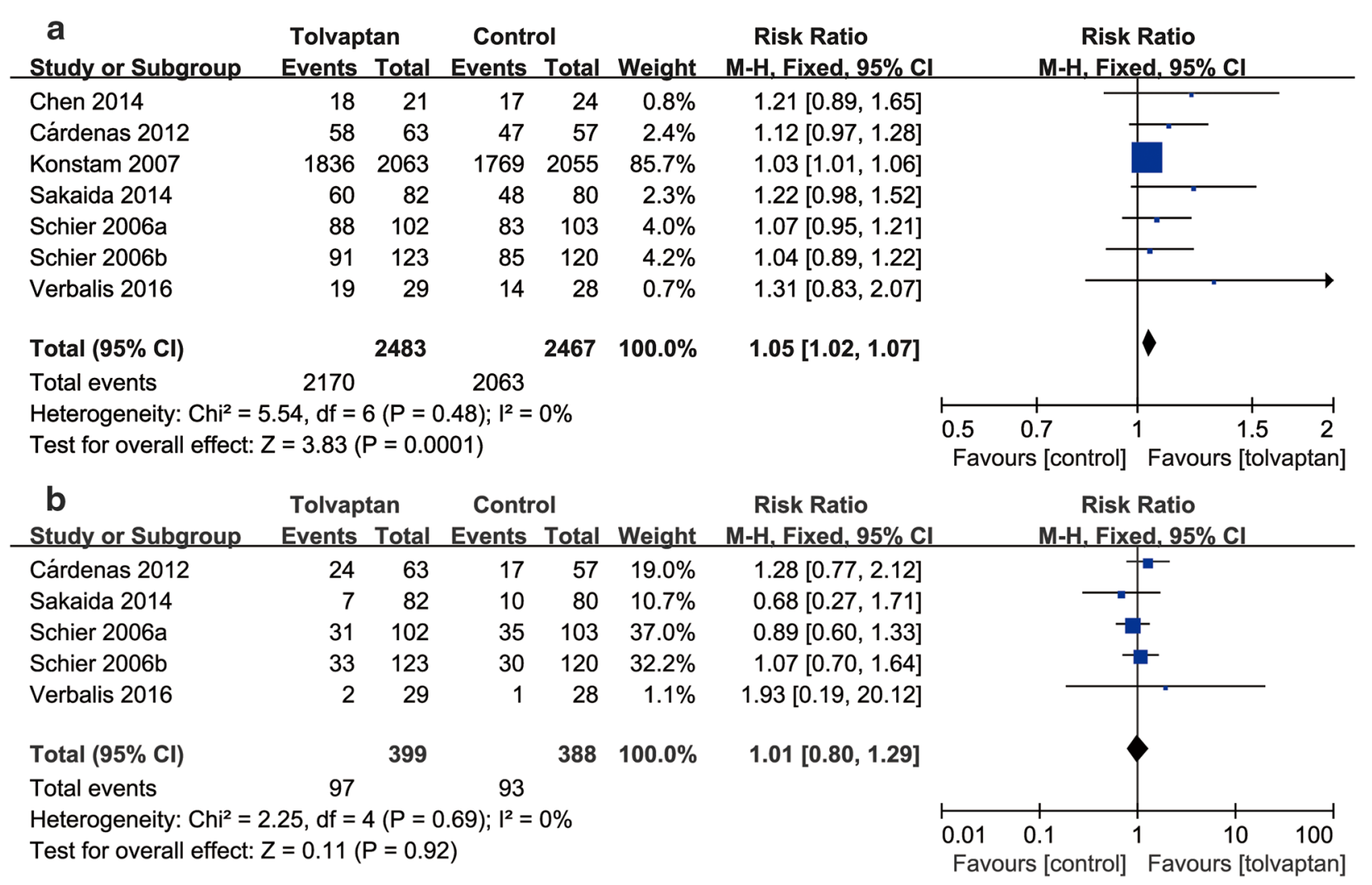

Fig. 5 Impact of tolvaptan on adverse event rates (a) and serious adverse event rates (b) in patients with hyponatremia. $C I$ confidence interval, $d f$ degrees of freedom, $M-H$ Mantel-Haenszel

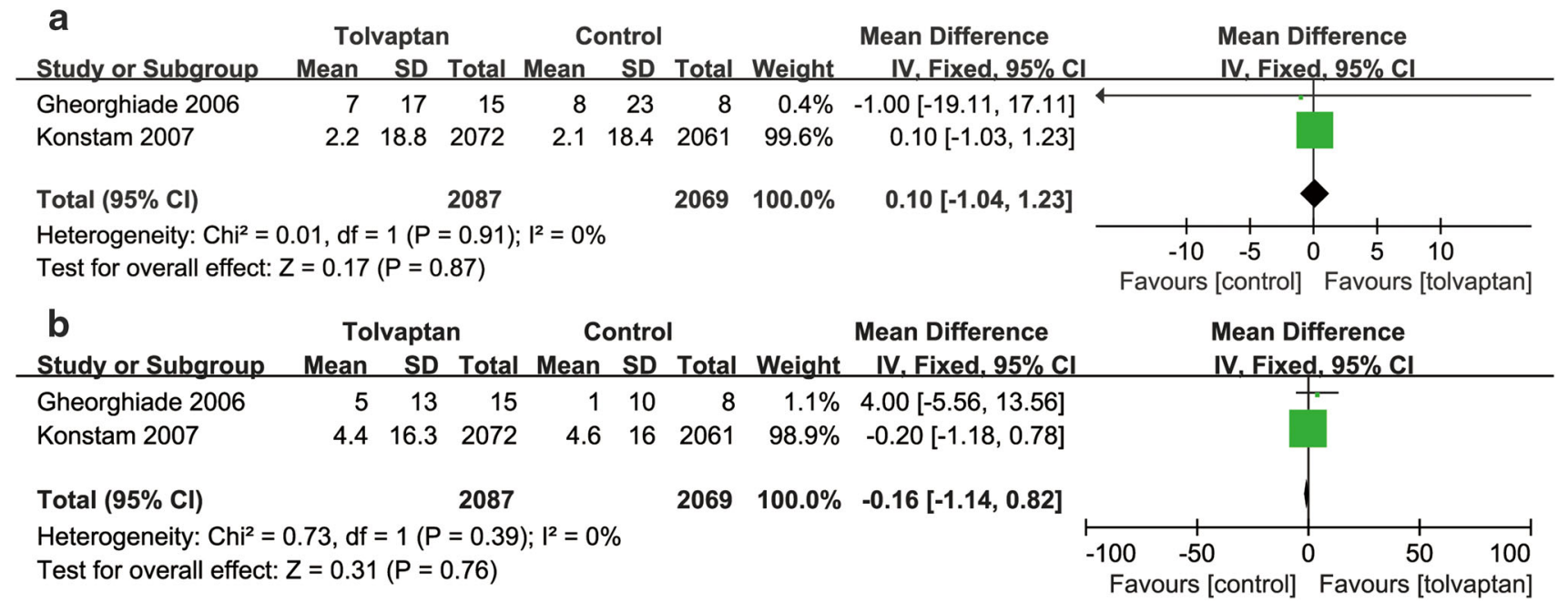

Fig. 6 Impact of tolvaptan on changes in systolic blood pressure (a) and changes in heart rate (b) in patients with hyponatremia. $C I$ confidence interval, $d f$ degrees of freedom, $I V$ inverse variance, $S D$ standard deviation 